\title{
Mechanismen der Autoimmunität
}

\author{
Mechanisms of Autoimmunity
}

Autoren

Institut
M. Kasperkiewicz, D. Zillikens

Klinik für Dermatologie, Allergologie und Venerologie, Universitätsklinikum Schleswig-Holstein, Campus Lübeck

\section{Bibliografie}

Dol $10.1055 / \mathrm{s}-2006-944623$

Akt Dermatol 2006; 32;

413-417 @ Georg Thieme

Verlag KG Stuttgart · New York ISSN 0340-2541

Korrespondenzadresse Dr. Michael Kasperkiewicz Klinik für Dermatologie, Allergologie und Venerologie Universitätsklinikum SchleswigHolstein, Campus Lübeck Ratzeburger Allee 160 23538 Lübeck Michael.Kasperkiewicz@uksh.de

\section{Zusammenfassung \\ $\nabla$}

Autoimmunität entsteht als Folge verschiedener, sich gegenseitig beeinflussender Faktoren, die insgesamt ein Versagen oder den Zusammenbruch der Autotoleranz verursachen. Durch Verwendung molekularbiologischer Techniken ist das Wissen über die Mechanismen von Autoimmunerkrankungen in den letzten Jahren gestiegen. Dies betrifft im Bereich der Dermatologie insbesondere die Kollagenosen und blasenbildenden Autoimmundermatosen. Bei den meisten Autoimmunerkrankungen besteht eine Assoziation mit bestimmten MHC Klasse I- und II-Mole-

\section{Einleitung}

\section{$\nabla$}

Eine zentrale Eigenschaft des Immunsystems ist seine Fähigkeit, zwischen körpereigenen und fremden Antigenen unterscheiden zu können. Der Entwicklung von Autoimmunerkrankungen liegt ein Zusammenbruch oder eine Umgehung der Selbsttoleranz zugrunde. Während Fortschritte der Molekularbiologie in den letzten Jahren zur Aufklärung der Struktur zahlreicher Zielantigene beitrugen, ist die Ursache der Autoantikörperbildung immer noch weitgehend unklar. Diskutiert werden unter anderem genetische, immunologische, infektiöse und medikamentöse Mechanismen ( Abb. 1). Das Spektrum der Autoimmunerkrankungen ist vielgestaltig, wobei die Haut bei systemischen Autoimmunkrankheiten (z. B. Kollagenosen), bei organspezifischen Autoimmunerkrankungen anderer Organe (z.B. prätibiales Myxödem beim M. Basedow) und bei Erkrankungen, die durch Autoantikörper gegen Strukturproteine der Haut hervorgerufen werden (blasenbildende Autoimmundermatosen), betroffen sein kann ( $\bullet$ Tab. 1). külen. Die immunologischen Mechanismen, welche die Autoimmunität beeinflussen, beinhalten Störungen der Lymphozytenselektion und peripherer Toleranzvorgänge, polyklonale Aktivierung autoreaktiver Lymphozyten, Kreuzreaktionen zwischen Antigenen und Störungen in der Regulation von Lymphozytenreaktionen. Infektionen, Medikamente, Gewebeschädigungen, hormonale Faktoren, UV-Licht und Malignome tragen ebenfalls zum Entstehen von Autoimmunerkrankungen bei. Das verbesserte Verständnis der Pathomechanismen der Autoimmunität lässt für die Zukunft neue immunmodulatorische Therapieansätze erhoffen.

\section{Genetische Faktoren \\ $\nabla$}

Als mögliche Voraussetzung für die Entwicklung einer Autoimmundermatose wurde bei einigen Krankheitsbildern eine erhöhte genetische Suszeptibilität der Patienten nachgewiesen (Übersicht in [1]). Bezüglich der Prädisposition für Autoimmunität richtet sich das Hauptinteresse derzeit auf die MHC-Gene. Hierzu werden mehrere Hypothesen diskutiert. (a) Die erste berücksichtigt die Rolle von MHC-Molekülen beim Aufbau des individuellen Antigen-spezifischen T-Zell-Repertoires während der Reifung im Thymus. Die Anwesenheit eines speziellen Haplotyps könnte infolge positiver und negativer Selektionsprozesse zum Überleben autoreaktiver T-Lypmphozyten führen und somit zur Störung der immunologischen Toleranz prädisponieren. (b) Eine zweite Hypothese zielt auf die MHC-Moleküle als wichtige Strukturen bei der Selektion und Präsentation von Peptiden durch Antigen-präsentierende Zellen (APCs). Abhängig von Größe, Form und biochemischen Eigenschaften des am distalen Ende des MHC-Moleküls gelegenen Peptidspaltes können bei der Selektion von antigenen Epitopen im Rahmen der Antigenpräsentation durch APCs 


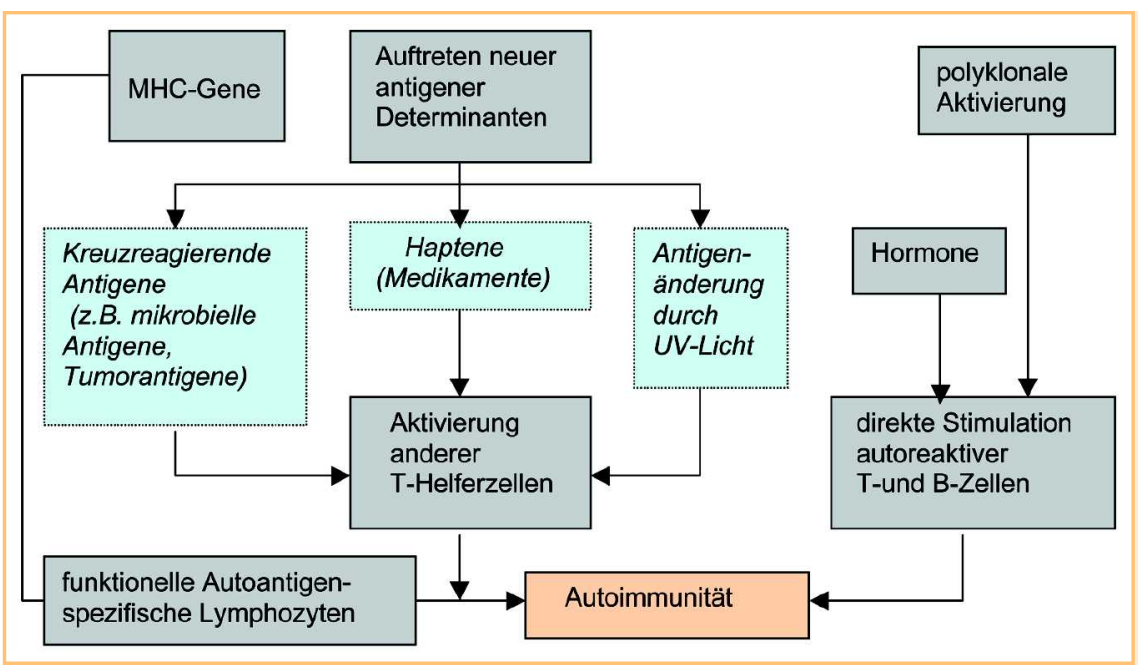

Abb. 1 Mögliche Induktion von Autoimmunität. Neben genetischen Faktoren, die eine gewisse individuelle Prädisposition bedingen, können unter anderem auch immunologische Faktoren, die z.T. auch genetische Ursachen haben, sowie mikrobielle, medikamentöse, hormonale und Umweltfaktoren eine Autoimmunität hervorrufen.

Tab. 1 Autoantikörper bei dermatologischen Erkrankungen

\begin{tabular}{ll}
\hline $\begin{array}{l}\text { Kollagenosen } \\
\text { SLE }\end{array}$ & Spezifität der Autoantikörper \\
\hline $\begin{array}{l}\text { SCLE, Sjögren-Syndrom, } \\
\text { neonatales LE-Syndrom }\end{array}$ & Ro/La \\
\hline $\begin{array}{l}\text { Medikamenten- } \\
\text { induzierter LE }\end{array}$ & Histone H2A, H2B \\
$\begin{array}{l}\text { Mixed connective } \\
\text { tissue disease }\end{array}$ & U1 RNP \\
\hline $\begin{array}{l}\text { CREST-Syndrom } \\
\text { Diffuse systemische } \\
\text { Sklerodermie }\end{array}$ & Antizentromer \\
\hline $\begin{array}{l}\text { Dermatomyositis- } \\
\text { Sklerodermie-overlap }\end{array}$ & Scl-70 \\
\hline $\begin{array}{l}\text { Poly(Dermato-)myositis } \\
\text { bullöse Autoimmun- } \\
\text { dermatosen }\end{array}$ & PM-Scl \\
\hline $\begin{array}{l}\text { Pemphigus vulgaris } \\
\text { Pemphigus foliaceus }\end{array}$ & Jo-1 \\
\hline $\begin{array}{l}\text { Medikamenten-induzierter } \\
\text { Pemphigus }\end{array}$ & Spezifität der Autoantikörper \\
\hline Paraneoplastischer Pemphigus & Desmoglein 3, 1, Desmocollin \\
\hline $\begin{array}{l}\text { Bullöses Pemphigoid } \\
\text { Schleimhautpemphigoid }\end{array}$ & Desmoglein 1 \\
\hline $\begin{array}{l}\text { Pemphigoid gestationis } \\
\text { Lineare IgA-Dermatose }\end{array}$ & Periplakin, Envoplakin; Plectin, \\
\hline Epidermolysis bullosa acquisita & BP 230, 170 kD-Protein \\
\hline Büser SLE & BP 180, BP 230 180, BP 230 \\
\hline Typ VII Kollagen \\
\hline Germatis herpetiformis & $\begin{array}{l}\text { Gewebstrasglutaminase, Gluten, } \\
\text { Gliadin, Endomysium }\end{array}$ \\
\hline
\end{tabular}

verstärkende oder hemmende Effekte ausgelöst werden. Im Tierversuch kann man so durch Experimente, bei denen durch Mutation die distale Aminosäuresequenz des MHC-Peptids verändert wird, die Selektion und Präsentation von antigenen Epitopen an autoreaktive T-Zellen beeinflussen. (c) Die dritte Hypothese identifiziert die MHC-Moleküle selbst mit den Autoantigenen. Die Durchbrechung der Toleranz geschieht hierbei als Folge der Ähnlichkeit von Aminosäuresequenzen der HLA-Moleküle mit antigenen Peptiden infektiöser Herkunft (molecular mimicry). So könnte eine normale immunologische Antwort auf exogene Peptide in ein Autoimmungeschehen gegen eigene HLA-Deter- minanten wechseln. MHC-Gene bestimmen somit in entscheidendem Maße über Krankheitsresistenz und Empfänglichkeit sowie über chronisch veränderte Immunantworten [2].

Bei den Kollagenosen wurde z.B. für den SLE unter Kaukasiern eine vermehrte Assoziation mit HLA-DR3 und -DR2 beschrieben. Es bestehen auch Hinweise für MHC-Assoziationen mit bestimmten Verläufen und distinkten Autoantikörperspezifitäten, wobei Anti-Ro-Antikörper vermehrt bei DR3-positiven Haplotypen mit Hautbeteiligung und hoher CD4/CD8-Ratio vorkommen, während DR2-Haplotypen vermehrt Anti-ds-DNS Antikörper bilden, zur Nierenbeteiligung neigen und eine eher erniedrigte CD4/CD8-Ratio aufweisen $[3,4,5]$.

Auch bei den bullösen Autoimmundermatosen besteht in einigen Fällen ein gehäuftes Vorkommen mit bestimmten HLA-Mustern und ein Zusammenhang mit autoreaktiven T-Lymphozyten. Auf eine Beteiligung von CD4+ T-Lymphozyten z. B. in der Pathogenese des Pemphigus vulgaris weist die starke Assoziation mit HLA-DR $\beta 1^{*} 0402$ und HLA-DQ $\beta 1^{*} 0503$ hin [6]. Für den häufigsten Vertreter der bullösen Autoimmundermatosen, dem bullösen Pemphigoid, wurden ebenfalls autoreaktive T-Helfer-Zellen nachgewiesen. Interessanterweise soll bei dieser Erkrankung die Epitop-Erkennung ebenfalls durch bestimmte HLA-II-Allele, v.a. HLA-DQ $\beta 1^{*} 0301$, eingeschränkt sein $[7,8]$. Die Tatsache, dass die mit Autoimmunität assoziierten MHC-Haplotypen auch in hoher Frequenz bei gesunden Individuen gefunden werden, liegt darin begründet, dass mehrere genetische Loki involviert sind und andere Faktoren eine zusätzliche Rolle spielen.

\section{Toleranzmechanismen}

$\nabla$

Um Autoimmunität zu verhindern sind Prozesse wichtig, die sicher stellen, dass unter den Lymphozyten, die sich in den zentralen lymphatischen Organen zur Reife differenzieren, möglichst keine autoreaktiven Klone vorhanden sind (zentrale Toleranz). Autoreaktive Lymphozyten, die diesem Selektionsprozess entgehen und funktionelle Reife erlangen, können auch zu einem späteren Zeitpunkt, in der Peripherie, inaktiviert werden (periphere Toleranz). 


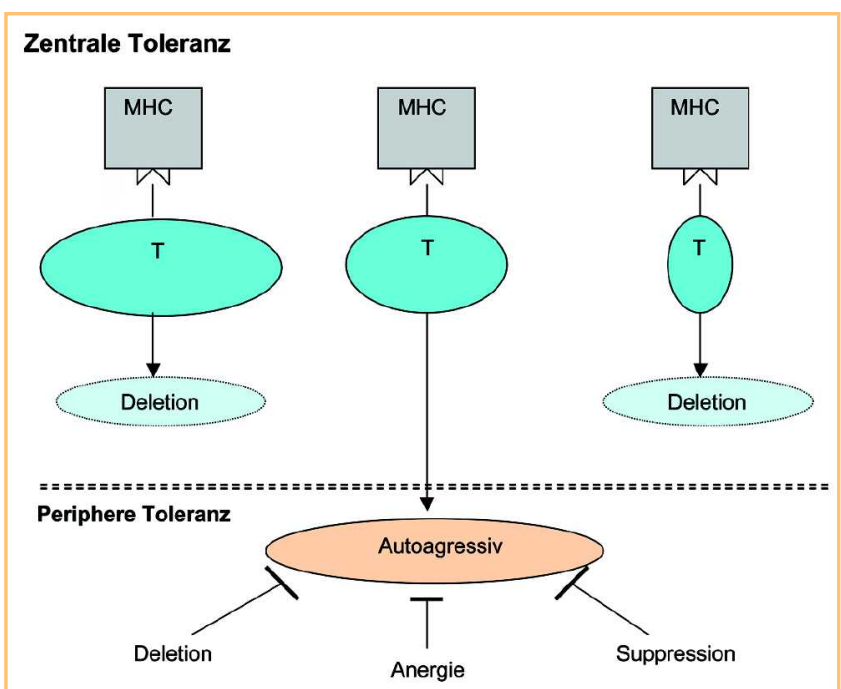

Abb. 2 Zentrale und periphere Toleranzmechanismen. Zentrale Toleranz: Im Thymus werden T-Zellen mit sehr hoher bzw. niedriger Affinität des T-Zellrezeptors für MHC-Moleküle durch aktive Deletion eliminiert. T-Zellklone mit intermediärer Affinität erfahren eine positive Selektion und gelangen in die Peripherie. Periphere Toleranz: In die Peripherie ausgewanderte T-Zellen können hier noch durch Deletion, Anergie und Suppression beseitigt werden.

\section{Zentrale Toleranz}

Die Deletion autoreaktiver T-Zellen ist der Hauptmechanismus der T-Zelltoleranz. Körpereigene Proteine werden verarbeitet und in Assoziation mit eigenen MHC-Molekülen auf antigenpräsentierenden Zellen des Thymus präsentiert. T-Lymphozyten, deren Rezeptoren eine zu geringe Affinität für diese Peptid/ MHC-Komplexe aufweisen, erhalten keine positiven Signale zum Überleben. Andere T-Zellen mit sehr hoher Affinität werden zur Apoptose veranlasst, und dieser Prozess wird als sog. negative Selektion bezeichnet. Die restlichen Zellen mit intermediärer Affinität ihres Rezeptors reifen im Thymus und emigrieren schließlich als potenziell autoreaktive Lymphozyten ins Blut (positive Selektion). Nicht alle Antigene kommen im Thymus vor und führen zur T-Zellselektion, deshalb sind periphere Mechanismen notwendig, um die Entwicklung autoimmunologischer Prozesse zu verhindern (Übersicht in [9]) ( $\bullet$ Abb. 2).

\section{Periphere Toleranz}

Sowohl Deletion, Anergie als auch Suppression werden als Sicherheitsmechanismen zum Aufrechterhalten der Autotoleranz in den Fällen postuliert, in denen es einigen autoreaktiven T-Zellen gelingt, der negativen Selektion zu entweichen und in die Peripherie zu gelangen.

Deletion kommt v.a. im Zusammenhang mit einem plötzlichen Auftreten hoher Antigenmengen vor, z.B. bei Verbrennungen und viralen Infektionen der Haut oder nach Injektion von Superantigenen [10,11]. Die Deletion autoreaktiver T-Zellen wird durch apoptotische Prozesse vermittelt und involviert den Apoptose-induzierenden Fas-Rezeptor (CD95) und seinen Liganden (FasL). Sowohl Fas als auch FasL wird auf aktivierten T-Zellen exprimiert, so dass die Interaktion dieser beiden Moleküle zur Apoptose führen kann.

Anerge T-Zellen sind dadurch charakterisiert, dass diese nach Antigenerkennung kein IL-2 produzieren und somit nicht vollständig aktiviert werden bzw. deutlich weniger proliferieren.
Dabei können unterschiedliche Faktoren zur Anergieinduktion in T-Zellen führen:

1. Fehlen des zweiten kostimulatorischen Signals. Aktivierte T-Lymphozyten exprimieren CD152 (CTLA-4). Die Interaktion von CD152 mit auf antigenpräsentierenden Zellen exprimierten kostimulatorischen Molekülen CD80 und CD86 hat eine Inhibition der T-Zellaktivierung zur Folge, und eine CD152-Blockade begünstigt die Entwicklung verschiedener Autoimmunerkrankungen im Mausmodell [12,13].

2. „Altered peptide ligands“. Der T-Zell-Rezeptor einer autoreaktiven Zelle wird normalerweise durch seinen Ligand-MHCKomplex voll stimuliert. Eine Änderung des immundominanten Selbst-Epitops führt zu einem „altered peptide ligand“, der nach Interaktion mit der autoreaktiven T-Zelle neben einer Verschiebung des Th1/Th2 Zytokinprofils auch zur Anergie der T-Lymphozyten führen kann [14].

3. Direkte Beeinflussung von dendritischen Zellen. Dendritische Zellen stellen die potentesten antigenpräsentierenden Zellen dar und sind neben ihrer Rolle bei der Immunabwehr gegen Fremderreger ein wichtiger Faktor bei der Auslösung von Autoimmunerkrankungen durch Präsentation von Autoantigenen an autoreaktive T-Zellen [15]. Durch Behandlung mit entsprechenden Zytokinen bzw. Zytokinkombinationen können dendritische Zellen vom immunogenen in einen tolerogenen Stand gebracht werden. Z. B. können humane dendritische Zellen nach Behandlung mit IL-10 sowohl in $\mathrm{CD}^{+}$- als auch CD8+-T-Zellen eine alloantigen- oder peptidspezifische Anergie induzieren, die zusätzlich Suppressoreigenschaften besitzen $[16,17]$. Die Bildung sog. regulatorischer T-Zellen gehört zum dritten Mechanismus der peripheren Toleranz und beruht auf der Fähigkeit, die Aktivierung anderer T-Lymphozyten und antigenpräsentierender Zellen zu supprimieren (Übersicht in $[18,19])$. Eine weitere Population stellen CD4 ${ }^{+}$ /CD25+-T-Zellen dar, die sowohl bei der Maus als auch bei Menschen nicht-antigenspezifische, regulatorische Effekte haben [20]. Dabei könnten CD152, TGF- $\beta$ und IL-10 zur Kontrolle autoreaktiver T-Zellen beitragen [21,22,23]. Die regulatorische Funktion von Th-Zellen wird wahrscheinlich durch unreife oder inkomplett gereifte dendritische Zellen initiiert [23].

\section{Die Rolle von Mikroorganismen \\ $\nabla$}

Potenziell autoreaktive Lymphozyten, die den Mechanismen der zentralen und peripheren Toleranz entgangen sind, können durch unterschiedliche „Trigger“ aktiviert werden und zu Autoimmunerkrankungen führen. Für zahlreiche dermatologische Autoimmunerkrankungen werden Infekte als auslösende Ursachen angesehen. So ist beispielsweise die eruptive Psoriasis vulgaris häufig mit einer Streptokokkenpharyngitis, der brasilienische Pemphigus foliaceus möglicherweise mit einem vektorübertragenden viralen Faktor und der murine SLE eventuell mit Retroviren und ihren Genprodukten assoziiert $[24,25,26]$. Unterschiedliche Mechanismen könnten dabei für eine Autoimmunreaktion verantwortlich sein:

1. Molekulare Mimikry ist die populärste Hypothese. Mikrobielle Antigene mit Homologie zu körpereigenen Antigenen führen dabei durch Umkehr der Immunantwort gegen das eigentliche infektiöse Agens in eine Autoimmunreaktion gegen das immunologisch ähnliche Selbst-Peptid. 
2. Beim antigenunspezifischen Modell der Bystander-Aktivierung werden autoreaktive T-Zellen, die für das Autoantigen $X$ spezifisch sind, während einer z.B. gegen das virale Antigen Ygerichteten Immunantwort aktiviert. Der durch die Entzündung herbeigeführte Gewebeschaden könnte zur Freilegung von kryptischen Autoantigenen führen, die dann den autoreaktiven Zellen präsentiert werden. Außerdem entsteht durch die Entzündung ein lokales Milieu mit der Folge einer vermehrten Expression von MCH-Molekülen bzw. verstärkter Zytokinsekretion und schließlich der Aktivierung von „Bystander"-T-Zellen.

3. Einen weiteren Mechanismus stellen Superantigene dar. Diese durch viele Pathogene produzierten Peptide können durch Bindung außerhalb des T-Zellrezeptors und der MHC-Peptidbindungsgrube zur Aktivierung von Lymphozyten führen. Diese Peptide sind somit nicht auf MHC-Moleküle beschränkt, werden nicht durch antigenpräsentierende Zellen prozessiert und sind nicht von spezifischer T-Zell-Erkennung abhängig, zumal sie an nicht polymorphe, variable T-Zell V $\beta$ Ketten binden. Superantigene, die üblicherweise mehr als eine $\mathrm{V} \beta$-Kette erkennen, können im Vergleich zur spezifischen Peptiderkennung mehr als 100000-mal mehr T-Lymphozyten aktivieren. Bei einer Infektion könnten somit Superantigene nicht nur polyklonale T-Zellen, sondern auch die potenziell autoreaktiven Zellen aktivieren und die immunologische Toleranz durchbrechen.

\section{Induktion durch Medikamente \\ $\nabla$}

Geringfügige Veränderungen in der Antigenstruktur können zu einem Toleranzverlust und damit zu einer gegen körpereigene Epitope gerichteten Immunreaktion führen. Neoantigene entstehen zum Beispiel bei medikamenteninduzierter Autoimmunität. Das Medikament bindet an autologe Polypeptide und verändert diese derart, dass sie in veränderter, nicht mehr tolerogener Konformation präsentiert werden. Zusätzlich kann das Medikament eine normale Antigenverarbeitung verhindern und dadurch eine Präsentation eines normalerweise nicht präsentierten Peptides bewirken, z.B. statt der Sequenz aa 780-800 eines Proteins, gegen das Toleranz besteht, würde das Peptid aa 850-860 präsentiert, gegen das keine Toleranz besteht.

Auf dem Gebiet der Dermatologie konnten verschiedene Medikamente als Kofaktoren für die Auslösung von Autoimmunkrankheiten identifiziert werden. Meist bilden sich die klinischen Symptome nach Absetzen zurück, können aber bei Wiedereinnahme rezidivieren. Bei den bullösen Autoimmundermatosen der Pemphigusgruppe können sulfhydrylhaltige Medikamente vermutlich direkt mit Desmosomen interferieren und zur Störung der Bindung desmosomaler Proteine führen. Lediglich nach Einnahme von Penicillamin ist medikamenteninduzierter Pemphigus nennenswert häufig und tritt bei ca. 7\% der über 6 Monate behandelten Personen auf, wobei es sich dann überwiegend um einen Pemphigus foliaceus handelt [27]. Daneben liegen Berichte über eine medikamentöse Induktion von bullösem Pemphigoid (Furosemid), Schleimhautpemphigoid (Penicillamin) und linearer IgA-Dermatose (Vancomycin) vor $[27,28,29]$. Innerhalb der Gruppe der Kollagenosen sind Hydralazin, Procainamid und Isoniazid für ihre SLE-induzierende Fähigkeit bekannt, wobei neben einem gestörten Medikamentenmetabolismus (langsamer Acetylierungsstatus) Interaktionen mit DNS, Immunzellen oder Komplement mögliche Mechanismen darstellen [30].

\section{Andere Faktoren \\ $\nabla$}

Neben den bereits erwähnten Vorgängen als auslösende Mechanismen können verschiedene andere Bedingungen als Kofaktoren für die Auslösung von Autoimmunerkrankungen eine wichtige Rolle spielen. Die Bedeutung von Hormonen geht aus einer Vielzahl von empirischen Beobachtungen hervor und trifft vor allem für den SLE zu: die Gynäkotropie, die Verschlechterung durch Schwangerschaft und Östrogenzufuhr sowie die erhöhten Spiegel des stark feminisierenden Östrogenmetaboliten 16-Hydroxyöstron bei Patientinnen mit Autoimmunerkrankungen [2]. Auch kann UV-Licht, möglicherweise durch Änderungen der Zellkernantigenität, die zu erhöhter Immunogenität führt, SLESchübe auslösen [31]. Eine Induktion durch Malignome ist z.B. für den paraneoplastischen Pemphigus beschrieben, wobei als Ursache Kreuzreaktivitäten von Autoantikörpern, die gegen Tumorantigene gerichtet sind, mit desmosomalen Strukturen vermutet werden oder die Bildung autoreaktiver Lymphomzellklone [32].

\section{Ausblick}

$\nabla$

Der Einsatz neuer Untersuchungsmethoden und das zunehmende Wissen über Autotoleranz haben in den letzten Jahren auch zu neuen Erkenntnissen über die Induktion von Autoimmunität geführt. Weitere Untersuchungen zur Identifizierung von Antigenen und Toleranzmechanismen könnten neben der Klärung pathophysiologischer Faktoren auch Wege zu spezifischeren und nebenwirkungsärmeren Therapiekonzepten eröffnen. Diese Strategien dürften unter anderem Vakzinierungen und die Induktion immunologischer Toleranz unter Verwendung rekombinanter Formen der Autoantigene einschließen.

\section{Abstract}

\section{Mechanisms of Autoimmunity \\ $\nabla$}

Autoimmunity originates as a result of different, mutually influencing factors together causing the failure or breakdown of selftolerance. By use of biomolecular techniques, knowledge of the mechanisms of autoimmune diseases has extended during the recent years. In dermatology, this concerns in particular connective tissue and autoimmune bullous diseases. For most autoimmune diseases, an association exists with certain MHC class I and II molecules. The immunological mechanisms underlying autoimmunity include disturbances in the selection of lymphocytes and peripheral tolerance processes, polyclonal activation of autoreactive lymphocytes, cross-reactions between antigens and failure in the regulation of lymphocytic reactions. Infections, drugs, tissue damages, hormonal factors, ultraviolet light and malignomas may also contribute to the induction of autoimmune diseases. Increasing knowledge on the pathomechanisms of autoimmunity, may lead to novel immunomodulatory strategies in the future. 


\section{Literatur}

1 Theofilopoulos AN, Kono DH. The genes of systemic autoimmunity. Proc Assoc Am Physicians 1999; 111: 228 - 240

2 Winchester RJ, Lahita RG. Genetic susceptibility to systemic lupus erythematosus.In: Lahita RG (ed)Systemic Lupus erythematosus. New York Wiley Medical Publication; 1987: 81 - 118

3 Mills JA. Systemic lupus erythematosus. New Engl J Med 1994; 330: $1871-1879$

4 Olsen ML, Arnett FC, Reveille JD. Contrastic molecular patterns of MHC class II allels associated with anti-Sm and anti-RNP precipitin autoantibodies in systemic lupus erythematosus. Arthr and Rheum 1993; 36: $94-104$

5 Smolen IS, Morimoto Ch, Steinberg AD, Wolf A, Schlossmann SF, Steinberg RT, Penner E, Reinherz E, Reichlin M, Chused TM. Systemic lupus erythematosus: Delineation of subpopulations by clinical, serology and T-cell subset analysis. Amer J med Sci 1985; 289: 139-147

6 Hertl M, Riechers R. Analysis of the T cells that are potentially involved in autoantibody production in pemphigus vulgaris. J Dermatol 1999; 26: $748-752$

7 Büdinger L, Borradori L, Yee C, Eming R, Ferencik S, Grosse-Wilde H, Merk $H F$, Yancey $K$, Hertl M. Identification and characterization of autoreactive $T$ cell responses to bullous pemphigoid antigen 2 in patients and healthy controls. J Clin Invest 1998; 102: $2082-2089$

8 Lin MS, Gharia MA, Swartz SJ, Diaz LA, Giudice GJ. Identification and characterization of epitopes recognized by $T$ lymphocytes and autoantibodies from patients with herpes gestationis. J Immunol 1999; 162: $4997-4999$

9 Kamradt T, Mitchison NA. Tolerance and autoimmunity. N Engl J Med 2001; 344: 655-664

10 Webb S, Morris C, Sprent J. Extrathymic tolerance of mature T cells: clonal elimination as a consequence of immunity. Cell 1990; 63: $1249-1256$

11 Moskophidis D, Lechner F, Pircher H, Zinkernagel RM. Virus persistence in acutely infected immunocompetent mice by exhaustion of antiviral cytotoxic effector T cells. Nature 1993; 362: 758 - 761

12 Egen JG, Kuhns MS, Allison JP. CTLA-4: new insights into its biological function and use in tumor immunotherapy. Nat Immunol 2002; 3: $611-618$

13 Sharpe AH, Freeman GJ. The B7-CD28 superfamily. Nature Rev Immunol 2002; 2: 116- 126

14 Nicholson LB, Greer JM, Sobel RA, Lees MB, Kuchroo VK. An altered peptide ligand mediates immune deviation and prevents autoimmune encephalomyelitis. Immunity 1995; 3: 397-405

15 Ludewig B, Odermatt B, Ochsenbein AF, Zinkernagel RM, Hengartner $H$. Role of dendritic cells in the induction and maintenance of autoimmune diseases. Immunol Rev 1999; 169: 45 - 54
16 Steinbrink K, Wolfl M, Jonuleit H, Knop J, Enk AH. Induction of tolerance by IL-10-treated dendritic cells. J Immunol 1997; 159: 4772 - 4780

17 Steinbrink K, Jonuleit H, Muller G, Schuler G, Knop J, Enk AH. Interleukin10-treated human dendritic cells induce a melanoma-antigen-specific anergy in CD8(+) T cells resulting in a failure to lyse tumor cells. Blood 1999; 93: $1634-1642$

18 Sakaguchi S. Regulatory T cells: key controllers of immunologic selftolerance. Cell 2000; 101: 455-458

19 Maloy KJ, Powrie F. Regulatory T cells in the control of immune pathology. Nat Immunol 2001; 2: 816-820

20 Jonuleit J, Schmitt E, Stassen M, Tuettenberg A, Knop J, Enk AH. Identification and functional characterization of human $\mathrm{CD} 4{ }^{+} \mathrm{CD} 25^{+} \mathrm{T}$ cells with regulatory properties isolated from peripheral blood. J Exp Med 2001; 193: 1227-1238

21 Jonuleit H, Schmitt E, Kakirman H, Stassen M, Knop J, Enk AH. Infectious tolerance: human $\mathrm{CD} 25(+)$ regulatory T cells convey suppressor activity to conventional CD4(+) T helper cells. J Exp Med 2002; 196: 255 260

22 McHugh RS, Whitters MJ, Piccirillo CA, Young DA, Shevach EM, Collins M, Byrne MC. CD4(+)CD25(+) immunoregulatory T cells: gene expression analysis reveals a functional role for the glucocorticoid-induced TNF receptor. Immunity 2002; 16: 311 - 323

23 Shevach EM. CD4+CD25+ suppressor T cells: more questions than answers. Nat Rev Immunol 2002; 2: 389-400

24 Tervaert WC, Esseveld $H$. A study of the incidence of haemolytic streptococci in the throat in patients with psoriasis vulgaris, with reference to their role in the pathogenesis of this disease. Dermatologica 1970; 140: $282-290$

25 Crosby DL, Diaz LA. Endemic pemphigus foliaceus - Fogo Selvagem. Derm Clin 1993; 11: 453-462

26 Medstrand P, Lindeskog M, Blomberg J. Expression of human endogenous retroviral sequences in peripheral blood mononuclear cells of healthy individuals. J Gen Virol 1992; 73: 2463-2466

27 Ruocco V, Sacerdoti G. Pemphigus and bullous pemphigoid due to drugs. Int J Derm 1991; 30: 307-312

28 Ahmed AR, Kurgis BS, Rogers RS. Cicatricial pemphigoid. J Am Acad Derm 1991; 24: 987-1001

29 Collier PM, Wojnarowska F. Linear IgA disease and chronic bullous disease of childhood. Eur J Derm 1993; 3: 623-634

30 Schur PH. Clinical features of SLE.In: Kelley WN et al. (eds)Textbook of Rheumatology, 3rd ed.Philadelphia Saunders; 1989: 1101 - 1129

31 Golan TD, Elkon KB, Gharvi AE, Krueger JG. Enhanced membrane binding of autoantibodies to cultured keratinocytes of systemic-lupuserythematosus after ultraviolett $\mathrm{B} /$ ultraviolett $\mathrm{A}$ irridation. J Clin Immunol 1992; 90: 1067-1076

32 Camisa C, Helm TN. Paraneoplastic pemphigus is a distinct neoplasiainduced autoimmune disease. Arch Derm 1993; 129: 883-886 